\title{
Advantages of human umbilical vein scaffolds derived from cesarean section vs. vaginal delivery for vascular tissue engineering
}

\author{
Markus Hoenicka ${ }^{1 *}$, Volker R. Jacobs², Georgine Huber ${ }^{3}$, Franz- \\ Xaver Schmid ${ }^{1,4}$, Dietrich E. Birnbaum ${ }^{1,5}$
}

\begin{abstract}
${ }^{1}$ University Hospital of Regensburg, Department of Cardiothoracic Surgery, Regensburg, Germany

${ }^{2}$ Klinikum Rechts der Isar, Frauenklinik (OB/GYN), Technical University Munich, Germany

${ }^{3}$ University of Regensburg, Krankenhaus Barmherzige Brüder, Klinik St. Hedwig, Regensburg, Germany

${ }^{4}$ current address: Klinikum Krefeld, Department of Cardiothoracic Surgery, Krefeld, Germany

${ }^{5}$ current address: Kerckhoff-Klinik, Department of Cardiac Surgery, Bad Nauheim, Germany

*corresponding author. Phone: ++49-941-944-9901, Fax: ++49-941-944-9802, email: markus.hoenicka@klinik.uni-regensburg.de
\end{abstract}

Publication note:

Biomaterials. 2008 Mar;29(8):1075-84. Epub 2007 Nov 26.PMID: 18037164

DOI: $10.1016 /$ j.biomaterials.2007.11.010

http://www.elsevier.com/wps/find/journaldescription.cws home/30392/description\#de scription 


\begin{abstract}
The current study investigated whether the mode of delivery and the mode of sample collection affect the functional properties of umbilical veins as scaffolds for vascular tissue engineering purposes. HUV from planned c-sections (PCS) showed a 1.7-fold higher maximum contraction with potassium chloride compared to spontaneous vaginal deliveries (VD, $\mathrm{p}=0.029$ ). The maximum contractions with histamine were 2.0-fold and 2.9-fold higher in the PCS and emergency c-section (ECS) groups, respectively, compared to the VD group ( $\mathrm{p}=0.003)$. The dose-response curves of serotonin were shifted to the right approx. 6-fold and 5-fold in the VD group compared to PCS and ECS, respectively ( $p=0.009)$. There were no differences between the birth groups in terms of tetrazolium dye reduction, platelet adhesion, and the structural integrity. The release of the antithrombotic compound prostacyclin from vessels of the PCS and ECS groups was 6.6-fold and 3.5fold higher, respectively, than in the VD group $(\mathrm{p}<0.001)$. There was no correlation between the duration of ischemia and any of the functional parameters. This study provides evidence that vessels obtained from PCS are to be preferred for tissue engineering purposes, as they can be harvested in a sterile fashion and show superior vasoconstrictor responses and antithrombotic properties. The data also support a once-per-day pickup schedule for umbilical cords without a deterioration of the functional properties.
\end{abstract}

\title{
1. Introduction
}

Bypassing stenoses in coronary arteries requires small caliber grafts with unique properties. In contrast to vascular reconstructions in large-diameter vessels like aorta, the antithrombotic properties of the graft surface as well as its viscoelastic properties are of critical importance. To date, there is no clinically suitable bypass material based on synthetic polymers. Although endothelial seeding greatly improves the patency of e.g. PTFE grafts_[1], the compliance mismatch between grafts and coronary arteries remains to be solved_[2]. It is now widely recognized that extracellular matrix (ECM) would make an ideal scaffold for therapeutic applications_[3]. Deendothelialized human umbilical vein (HUV) has recently been suggested as a living scaffold for vas- 
cular tissue engineering [4]. Umbilical cords are a cheap and abundant source of stem cells and of vascular tissue, with recruitment rates exceeding $60 \%$ of all births in an ongoing study_[5]. Deendothelialized HUV contain ECM with suitable viscoelastic properties_[6] along with fetal smooth muscle cells which can synthesize and remodel ECM. Turning this semifinished biomaterial into a vascular graft will require two tissue engineering processes. First, in order to provide a hemocompatible and non-immunogenic surface, endothelial function has to be restored by seeding recipient-derived endothelial cells (EC). Second, the graft has to mature in a pulsatile bioreactor to create a wall structure and EC alignment suitable for the pressure and shear forces of the coronary circulation [7]. The quality of a vessel replacement engineered from umbilical vessels, and the difficulties associated with the engineering process, are likely to depend on the quality of the starting material. Therefore, the current study investigated the influence of the mode of birth on the functional properties of HUV.

The influence of the birth mode on the physiology of neonates has been investigated in several studies. It is generally accepted that delivery by a planned (elective) cesarean section (PCS) is less stressful for the fetus than a spontaneous vaginal delivery (VD). There is a body of evidence that PCS causes less oxidative stress as measured by the levels of lipid peroxidation products_[8,9] and of the antioxidant glutathione_[10] in the cord blood. Along the same lines, the levels of the stress indicators cortisol_[11], progesterone_[12], and norepinephrine [13] as well as the number of $\mathrm{CD}_{3} 4^{+}$mononuclear cells_[14] in the cord blood are lower after a PCS. This study tried to address the question whether the same differences affect the performance of HUV post partum.

With the exception of PCS, the exact time of birth, and thus the time of umbilical cord sample collection, is difficult to predict. Moreover, a large proportion of births occur at times which are inconvenient for a routine tissue engineering protocol. The situation is aggravated if, as it was the case in the present study, the samples have to be processed outside of the hospitals that provide them. This warranted an investigation whether the quality of HUV depends on the duration of ischemia. 


\section{Materials and Methods}

\subsection{Materials and Reagents}

Calcein AM was supplied by Molecular Probes (Eugene, OR, USA). Hank's Balanced Salt Solution (HBSS) was purchased from Invitrogen (Carlsbad, CA, USA), and fetal calf serum (FCS) was obtained from PAN (Aidenbach, Germany). All other reagents were of analytical grade and were purchased from Sigma (Taufkirchen, Germany) or from Merck (Darmstadt, Germany).

\subsection{Subjects and harvesting of umbilical cords}

A random and unselected group of pregnant women was recruited for the study [5] when they first reported to the maternity wards in the two participating medical centers, and written informed consent was obtained. The experiments were approved by the institutional review boards of both universities. A total of 40 VD, 19 PCS, and 18 ECS were included into the study. Patients with known contagious diseases like HIV or hepatitis were excluded.

The samples were divided into three groups according to the mode of delivery. The VD group contained all vaginal deliveries. The PCS group received all c-sections that were started before the onset of labor due to a medical indication or in response to the expectant mother's request. The ECS group received all c-sections that commenced after the onset of labor.

Samples of the umbilical cords originating from Klinik St. Hedwig were stored in Krebs-Henseleit buffer (KHB; NaCl $118 \mathrm{mM}, \mathrm{KCl} 4.7 \mathrm{mM}$, Mg$\mathrm{SO}_{4} 1.2 \mathrm{mM}, \mathrm{NaH}_{2} \mathrm{PO}_{4} 1.2 \mathrm{mM}, \mathrm{NaHCO}_{3} 16.7 \mathrm{mM}$, dextrose $5.5 \mathrm{mM}$, $\left.\mathrm{CaCl}_{2} \quad 1.2 \mathrm{mM}\right)$ supplemented with HEPES $(25 \mathrm{mM})$, penicillin $(100 \mathrm{U} / \mathrm{ml})$, and streptomycin $(100 \mu \mathrm{g} / \mathrm{ml})$ at $4^{\circ} \mathrm{C}$ immediately after parturition and transported to the lab. Cords originating from Klinikum Rechts der Isar were stored in HF-1 buffer (HBSS supplemented with HEPES $1 \mathrm{mM}$, penicillin $100 \mathrm{U} / \mathrm{ml}$, streptomycin $100 \mu \mathrm{g} / \mathrm{ml}$, and FCS 1\%). The minor difference in the storage solutions had logistic reasons only and did not affect the performance of the veins. In pilot transports a digital thermometer recorded the minimum and maximum temperatures inside the containers. The temperatures did not exceed $16^{\circ} \mathrm{C}$ during summer. 
Areas of the umbilical cords visibly damaged by clamps were excluded from the analysis. The HUV were dissected free from Wharton's Jelly, both to simplify handling and to properly model the situation of a tissue-engineered graft. The vessels were either cut into rings of $2 \mathrm{~mm}$ segment length for organ bath studies, or they were carefully opened longitudinally for functional studies of the vessel surface. Due to size constraints it was not possible to run each functional assay on every sample. Instead, the veins were randomly assigned to one or more assays until a sufficient number of data points were available per assay.

\subsection{Organ bath studies}

The tension of HUV rings was determined isometrically in an IOA-5300 organ bath system (FMI GmbH, Seeheim/Ober-Beerbach, Germany) filled with KHB under hypoxic conditions (5\% oxygen, $5 \%$ carbon dioxide, balance nitrogen) as described previously [4]. Four to eight rings of each HUV were used per experiment. In studies involving the relaxation of the vessels, one or two rings were randomly assigned to a time control group which did not receive the relaxing agent. The effect of relaxing agents was computed relative to these time controls to account for the spontaneous loss of tension over time.

\subsection{Histology}

HUV were fixed in phosphate-buffered paraformaldehyde (4\%) and embedded in paraffin. Thin sections were stained with Russell-Movat's Pentachrome procedure according to the manufacturer's protocol (American MasterTech Scientific, Lodi, CA, USA) to assess the appearance of smooth muscle, collagen, and elastic fibers as well as the condition of the endothelium.

\subsection{Scanning electron microscopy}

HUV were fixed in phosphate-buffered paraformaldehyde $(2 \%)$ supplemented with $2.5 \%$ glutardialdehyde and cut longitudinally into half or quarter segments. The samples were then dehydrated and sputtered with gold. Scanning electron micrographs were created on a Quanta400F scanning electron microscope (FEI, Hillsboro, OR, USA) using an accelerating voltage of $10 \mathrm{kV}$.

Scanning electron micrographs were evaluated by an independent reviewer in a blinded fashion. Overview images at 200x magnification 
were scanned for cracks and missing patches of endothelium. Each image was assigned an estimated percentage, in increments of $10 \%$, of intact endothelial coverage. Preparation artifacts, if any, were assumed to affect all groups to the same degree.

\subsection{Platelet adhesion assay}

The adhesion of human platelets to the luminal vessel surface was determined as described previously_[4]. In brief, longitudinally opened HUVs were mounted in tissue holders, which allowed access to a defined area $\left(0.33 \mathrm{~cm}^{2}\right)$ of the luminal surface, and incubated with a suspension of Calcein AM-labelled platelets of a known concentration. After an incubation at $37^{\circ} \mathrm{C}$ for $30 \mathrm{~min}$, the suspension was aspirated, and the wells were washed three times with phosphate-buffered saline. The adhering platelets were lysed using $1 \%$ Triton X-100, and the released amount of Calcein AM in the supernatants was determined fluorometrically. A calibration curve was constructed from a known amount of the same labelled platelets on polystyrene culture plates. The data are reported relative to the number of platelets per area adhering to polystyrene under the same conditions.

\subsection{Tetrazolium dye reduction}

The conversion of the chromogenic substrate 3-(4,5-dimethylthiazol-2yl)-5-(3-carboxymethoxyphenyl)-2-(4-sulfophenyl)-2H-tetrazolium (MTS, Promega, Madison, WI, USA) was determined on the luminal surface of longitudinally opened HUVs mounted in the same vessel holders as mentioned above and evaluated as described previously [4].

\subsection{Determination of prostacyclin release}

The luminal surfaces of HUVs mounted in vessel holders were incubated in an incubation buffer containing PBS supplemented with $5 \mathrm{mM}$ dextrose, $0.5 \mathrm{mM} \mathrm{MgCl}_{2}$, and $1.2 \mathrm{mM} \mathrm{CaCl}_{2}$ for $10 \mathrm{~s}, 1 \mathrm{~min}, 3 \mathrm{~min}$, $10 \mathrm{~min}$, and $15 \mathrm{~min}$ at $37^{\circ} \mathrm{C}$ to assess the basal release of prostacyclin. The supernatants were stored at $-80^{\circ} \mathrm{C}$ and analyzed using an enzymelinked immunosorbent assay kit according to the manufacturers protocol (Cayman Chemicals, Ann Arbor, MI, USA). The rates of prostacyclin release were determined by computing a linear regression fit to the average concentrations at each timepoint. 


\subsection{Data analysis and statistics}

Quantitative data are reported as mean \pm standard deviation. Half maximal effective concentrations $\left(\mathrm{EC}_{50}\right)$ and maximum response values were computed from the dose-response curves of each sample by fitting a Hill function. The means of the $\mathrm{EC}_{50}$ values were then computed for each birth mode. Differences in demographic data and in serotonin dose-response curves between birth modes were analyzed using ANOVA followed by the Kruskal-Wallis or the Holm-Sidak test as appropriate. Histamine dose-response curves were expressed as the ratio of the sample tensions and the donor-matched time control tensions to account for a time-dependent loss of tension. Within each birth mode, two-way repeated measurements ANOVA was used to detect a significant difference between histamine and control curves. In addition, sample curves were compared between birth modes using two-way ANOVA. Prostacyclin release rates were compared using two-way ANOVA, again followed by the Holm-Sidak test. Endothelial coverage was compared with the Mann-Whitney rank sum test. A statistically significant difference was assumed for $\mathrm{p}<0.05$.

\section{Results}

\subsection{Maternal and sample characteristics}

Table 1 shows the maternal characteristics as well as the physiologic parameters of the neonates. All but four births occurred at gestational ages of $37+0$ weeks or later. The four pre-term births were distributed evenly between VD and c-sections, and none of these pregnancies was shorter than 35+6 weeks. The duration of pregnancies terminated with a PCS was significantly shorter compared to the VD and ECS groups (ANOVA, $\mathrm{p}<0.001$ ), and the birth weights of the newborns were lower compared to the VD group (ANOVA, $\mathrm{p}=0.002$ ). The mean ages of mothers were not different between the modes of delivery (ANOVA, $p=0.054$ ), although the ages of mothers having an emergency c-section tended to be higher. The $\mathrm{pH}$ of the arterial umbilical cord blood was not significantly different between the groups (ANOVA, $\mathrm{p}=0.056$ ), but vaginal deliveries tended to result in a lower arterial $\mathrm{pH}$. The Apgar values at 5 and $10 \mathrm{~min}$ were significantly higher in vaginal deliveries (ANOVA, $\mathrm{p}<0.001)$. The duration of ischemia, measured as the time between birth and the start of the vessel preparation in the lab, was signific- 
antly longer in PCS compared to VD (ANOVA, $\mathrm{p}=0.043$ ).

\subsection{Effects of vasoactive compounds}

HUVs were contracted by depolarizing the smooth muscle with $150 \mathrm{mM} \mathrm{KCl} \mathrm{(Fig.} \mathrm{1).} \mathrm{The} \mathrm{maximum} \mathrm{tensions} \mathrm{of} \mathrm{HUVs} \mathrm{obtained} \mathrm{from}$ VD were significantly lower compared to those obtained from PCS (ANOVA, $\mathrm{p}=0.029$ ), reaching only $56.6 \%$ of the tension of the latter. To assess the receptor-mediated contraction, dose-response curves of the vasoconstrictor serotonin (5-hydroxytryptamine) were constructed (Fig. 2). The curves obtained from the c-section groups were found to differ significantly from the curves obtained from the VD group (two-way ANOVA, $\mathrm{p}<0.001)$. The $\mathrm{EC}_{50}$ values of HUVs obtained from PCS $(5.71 \mathrm{E}-9$, $\left.\log \mathrm{EC}_{50}=-8.24 \pm 0.62\right)$ and of HUVs obtained from ECS $(6.88 \mathrm{E}-9$, lo$\mathrm{gEC}_{50}=-8.16 \pm 0.26$ ) were significantly lower than of those obtained from $\operatorname{VD}\left(3.36 \mathrm{E}-8, \log \mathrm{EC}_{50}=-7.47 \pm 0.46\right.$, ANOVA, $\left.\mathrm{p}=0.009\right)$. The maximum tensions of both PCS and ECS vessel rings were not significantly higher compared to vessels from VD (ANOVA, $p=0.057$ ). Figure 3 shows that there was no correlation between ischemia time and maximum contraction by $\mathrm{KCl}\left(\mathrm{r}^{2}=0.027\right)$ or by serotonin $\left(\mathrm{r}^{2}=0.019\right)$ as well as between ischemia time and $\mathrm{EC}_{50}$ of serotonin $\left(\mathrm{r}^{2}=7.71 \mathrm{E}-3\right)$.

We assessed the endothelium-dependent relaxation of vessel rings by administering a concentration series of histamine after preconstriction with serotonin (Fig. 4). We observed no significant relaxation in any of the groups. However, all groups showed a significant vasoconstriction at histamine concentrations of $1 \mathrm{E}-6 \mathrm{M}$ and higher (ANOVA, $\mathrm{p}<0.05$ ). Vessels from PCS constricted significantly stronger than vessels from $\mathrm{VD}$ at histamine concentrations of $1 \mathrm{E}-6 \mathrm{M}$ and higher, whereas vessels from ECS showed a significantly stronger contraction compared to the VD group only at $1 \mathrm{E}-5 \mathrm{M}$ histamine (ANOVA, $\mathrm{p}=0.042$ ).

\subsection{Tetrazolium dye reduction}

The metabolic state of the vessels as measured by their capacity to reduce MTS was not significantly different (ANOVA, $\mathrm{p}=0.673$ ) between samples from PCS $\left(\mathrm{OD}_{490}=0.843 \pm 0.340, \mathrm{n}=7\right)$, from ECS $(0.915 \pm 0.327$, $\mathrm{n}=6)$, and from VD $(0.770 \pm 0.264, \mathrm{n}=9)$. There was also no correlation with the duration of ischemia $\left(r^{2}=0.019\right.$, Fig. 3). 


\subsection{Platelet adhesion}

There was no significant difference (ANOVA, $\mathrm{p}=0.912$ ) in the adhesion of platelets to HUV obtained from PCS $(0.177 \% \pm 0.094, n=3)$, from ECS $(0.178 \% \pm 0.046, \mathrm{n}=3)$, and from VD $(0.160 \% \pm 0.061, \mathrm{n}=5)$.

\subsection{Prostacyclin release}

The rates of prostacyclin release from the luminal faces of HUV were significantly higher in veins obtained from PCS and from ECS compared to veins obtained from VD (two-way ANOVA, $p<0.001$, Fig. 5). The rates were computed as $6.93 \mathrm{ng} \cdot \mathrm{ml}^{-1} \cdot \mathrm{min}^{-1}$ (correlation coefficient: 0.95, $\mathrm{n}=7$ ), $3.67 \mathrm{ng} \cdot \mathrm{ml}^{-1} \cdot \mathrm{min}^{-1}(0.96, \mathrm{n}=6)$ and $1.05 \mathrm{ng} \cdot \mathrm{ml}^{-1} \cdot \mathrm{min}^{-1}(0.95$, $\mathrm{n}=10$ ) for PCS, ECS, and VD, respectively.

\subsection{Vessel structure}

The evaluation of the luminal vessel surface by scanning electron microscopy revealed no significant difference (rank-sum test, $\mathrm{p}=0.944$ ) in endothelial coverage between PCS $(n=10)$ and VD $(n=4)$. The former resulted in a median of $80 \%$ coverage $(25 \%$ percentile: $60 \%, 75 \%$ percentile: $90 \%)$, whereas the latter resulted in $75 \%(55 \%, 95 \%)$. The histological images (Fig. 6) of HUV cross sections also demonstrated an intact endothelium and a mostly unstructured smooth muscle layer with a low content of elastic fibers. None of the vessel sections contained any signs of mechanical alterations, regardless of the mode of delivery.

\subsection{Correlations with other parameters}

The maximum contraction with potassium chloride, the $\mathrm{EC}_{50}$ values of serotonin, and the prostacyclin release were found to be significantly influenced by the birth mode. We also evaluated the influence of arterial $\mathrm{pH}$, gestational age, birth weight, age of the mother, sex of the baby, storage solution, and duration of ischemia on these functional properties. There were no significant differences between HUV from male and from female babies, or between veins stored in KHB and in HF-1 in terms of their functional properties (ANOVA). The correlation coefficients $r^{2}$ of all other correlations ranged between $2.34 \mathrm{E}-4$ and 0.143 and were considered too low to contribute to the observed differences. 


\section{Discussion}

To the best of our knowledge this is the first comprehensive study that investigates the impact of the different modes of birth on the function of HUV. Recent advances in tissue engineering [4] have turned HUV into a promising biomaterial. The compliance of HUV was shown to be comparable to human saphenous vein, one of the most common bypass materials [15]. Cryopreserved HUV could be combined with fresh or cryopreserved, recipient-matched endothelial cells_[16] and processed in a pulsatile bioreactor to create customized vascular grafts. This study tried to address the question if and how the mode of birth affects the functional properties of HUV and thus their utility as a biomaterial.

In general, PCS are considered less stressful for the fetus as compared to VD due to lower cord blood levels of the stress indicators cortisol, catecholamines, and $\beta$-endorphin_[17-22]. There is also evidence that vaginal deliveries are associated with an increased oxidative stress_[10] compared to c-sections. Therefore the working hyphothesis was that the lower level of stress in PCS will manifest itself beneficially in functional properties of HUV.

The maternal characteristics observed in the births that were included in our study are well in agreement with the long-term experiences in OB/GYN departments. The gestational age of births terminated by a PCS were significantly shorter compared to the other birth modes. This is a consequence of the definition of this group, as it contains all births before the onset of labor. This shorter duration of the pregnancies also explains the reduced birth weight. The only significant difference that is surprising at first is the longer duration of ischemia in PCS as compared to the other modes. All PCS except one were performed between 8:32 and 12:32 as they can usually be scheduled for the most convenient time of the day, whereas VD and ECS were distributed more randomly throughout the day. Thus the fixed pickup time in the morning skewed the distribution of ischemia times in favour of VD and ECS.

The reduction of tetrazolium dyes by membrane-bound enzymes is a marker for the redox state of cells and tissues_[23]. MTS is reduced mainly intracellularly and is thus linked to the mitochondrial metabolism, indirectly reflecting the potential to generate ATP [24]. We used the reduction of MTS as well as the contractile force to assess the pos- 
sible influence of the duration of ischemia on the vessel function. There was no correlation between ischemia time and MTS reduction, or between ischemia time and the contractions elicited by $\mathrm{KCl}$ or serotonin. Therefore it is safe to store umbilical cords for up to 40 hours under the conditions used in our study without a change of the redox state or of the contractile forces, which makes sample collection for tissue engineering purposes reasonably simple.

Sterility of the samples is crucial for their use as living scaffolds, as they cannot be sterilized like acellular scaffolds. Both storage solutions were supplied in sterile containers and included standard antibiotics to prevent bacterial growth. Visual and microscopic inspections of the storage solutions did not reveal any contaminations at the time of arrival in the lab. However, there is obviously no reliable way to obtain truly sterile umbilical cord samples from VD. On the other hand, planned c-sections are performed from the start within the sterile environment of a surgical theatre and while the amniotic sac is still intact, allowing the removal and storage of the umbilical cord under sterile conditions.

The birth modes differ in the amount of mechanical strain put on the umbilical cord. In c-sections the placenta is separated manually from the directly accessible uterus, whereas in vaginal births the delivery of the placenta may be assisted by gently pulling the remainder of the umbilical cord. However, umbilical cords are uniquely constructed to withstand both compression and elongation without harming the umbilical circulation [25], which makes mechanical alterations of the veins unlikely. The evaluation of the histological sections as well as of the scanning electron micrographs revealed no differences in the vessel structure and luminal surface, respectively, that would exceed the variation within the PCS group and thus could be seen as a consequence of any additional strain in the VD and ECS groups. The cross sections indicated an intact endothelium and an undisturbed smooth muscle layer. The minor endothelial damage seen in the en face scanning electron micrographs is likely due to preparation artifacts and was not different between birth modes. Therefore, umbilical cords from all three modes are equally suitable for tissue engineering purposes with regard to their structural integrity.

The vasomotor response of the veins were evaluated for two reasons. First, the vasoconstriction, along with the tetrazolium dye reduction, 
may serve as a measure for the functional integrity of the cells. Although long-term incubations in a bioreactor were outside the scope of the present study, it is reasonable to assume that undamaged cells will perform better in a tissue engineering scenario. Second, an appropriate vasomotor function is desirable in the finished graft, as it allows the vessel to respond to endogenous and pharmacological stimuli. HUV from VD showed a significantly lower maximum contraction in response to potassium chloride compared to PCS. The maximum contraction of ECS was intermediate. As the mitochondrial metabolism was unaffected by the mode of birth, it seems unlikely that the reduced maximum contraction is due to a lack of intracellular energy sources. We are aware that our experiments were not designed to distinguish between a temporary reduction of contractile force in response to an exhaustion of required factors and a more serious damage which would not allow a recovery. In any case, the differences persisted long enough to be detectable up to $40 \mathrm{~h}$ after birth.

Histamine acts as a vasorelaxant in some vascular beds due to an interaction with the endothelium[26], whereas higher doses of histamine cause a vasoconstriction by a direct interaction with the smooth muscle cells [27]. The maximum contractions in our experiments were significantly lower in vessels obtained from VD as compared to the csection groups. This indicates that the contractile capacity is diminished in the VD group, irrespective of the agent that causes the vasoconstriction.

Serotonin elicits a stronger vasoconstriction compared to thromboxane and norepinephrine in HUV [4]. This makes serotonin best suited to investigate the effects of the birth mode on receptor-mediated vasoconstriction. The $\mathrm{EC}_{50}$ values of both $\mathrm{c}$-section groups were significantly lower compared to the VD group. It is reasonable to assume that the values obtained from PCS best reflect the situation in utero, and that the values obtained from VD are caused by factors which are specific for this birth mode, like hour-long uterine contractions, stress, and maternal pain. In ECS, the fetus can suffer from the same amount of labor-induced stress with the exception that it does not pass the birth canal. Interestingly, there is no difference in the $\mathrm{EC}_{50}$ values of serotonin between the two c-section groups, although the duration of labour in ECS can be the same as in VD. The passage through the birth canal may therefore contribute to the factors that cause a desensitiza- 
tion of HUV towards serotonin, which is in accordance with the hypothesis that serotonin is involved in the closure of the umbilical circulation[28].

Contraction of HUV by serotonin is mediated by both $5-\mathrm{HT}_{2 \mathrm{~A}}$ and 5$\mathrm{HT}_{1 \mathrm{~B}}$ receptors_[29-31]. Cord blood serotonin levels were reported to be 2.8 times higher after VD compared to PCS in bovine_[32]. A desensitization of 5-HT receptors may thus explain the lower potency of serotonin in the VD group, although further studies with selective agonists and antagonists would have to reveal which of the receptor subtypes is involved.

Endogenous prostacyclin is both a vasodilator and a potent antithrombotic compound_[33,34] which relaxes umbilical arteries more efficiently than nitric oxide [35]. A sufficient prostacyclin release is obviously desirable in vessel grafts to provide for an antithrombotic surface. Our current data indicate a significantly higher rate of prostacyclin release from veins in both c-section groups compared to the VD group.

The medication of expectant mothers may influence the functional properties of the HUV as well and could potentially explain the differences that we observed between the birth modes. The medication was applied according to the individual requirements of each patient and thus was very heterogenous, both among patients within the same birth mode group and between the participating medical centers. The limited number of cases per substance did not allow a statistical evaluation. Instead we tried to identify medications 1 ) which have a mechanism of action that may interfere with the functional properties, 2) which are likely to cross the placenta, and 3) whose application shows a distribution between birth modes in the specific sets of experiments that parallels the observed differences. Of all substances administered to the patients in the last $24 \mathrm{~h}$ before birth, only metoclopramide (used to improve stomach motility) meets these criteria to hypothetically explain the results of the organ bath experiments. However, this substance has been described as a dopamine $\mathrm{D}_{2}$ receptor antagonist and a $5-\mathrm{HT}_{3}$ antagonist which does not affect placental vein contractions as these are mediated by the $5-\mathrm{HT}_{2}$ receptor_[36]. Oxytocin matches the pattern of the prostacyclin results if one assumes an inhibitory role. However, the substance is known to promote instead of inhibit prosta- 
cyclin release [37]. Within the limitations of this approach we consider it unlikely that the functional differences of HUV can be attributed to the medication of the expectant mothers.

Although we have shown in a previous study that HUV can be cryopreserved and seeded with EC_[4], the feasibility of a tissue engineering process based on this biomaterial needs to be evaluated in future studies. It remains to be shown whether the smooth muscle layer of HUV is capable of adapting to increasing pressure and shear forces either in a pulsatile bioreactor or in a xenogenic transplant model_[38]. However, as HUV consist of fetal tissue which continuously adapts to an increasing umbilical blood flow during pregnancy, growth and ECM synthesis should be at least comparable to tissue-engineered products which use adult recipient-derived smooth muscle cells or fibroblasts. Immunologically, a deendothelialized HUV is an allogeneic transplant, even when seeded with the recipient's EC. HUV have so far only been used as a glutaraldehyde-fixed graft for peripheral revascularization with good results_39]. However, these results cannot easily be transferred to a tissue-engineered graft based on deendothelialized HUV. The latter lacks the tanning process, which at least partially masks the antigenic sites, but will be covered by the recipient's own endothelium. Such an endothelium has been shown to establish full hemocompatibility of arterial allografts without immunosuppression with a patency of several years_[40]. To show that the same holds true for a HUV-based graft was beyond the scope of the current study.

\section{Conclusion}

This study has shown that a simplified sample collection protocol involving a once-per-day pickup does not negatively affect the performance of HUV as a starting material for tissue engineering purposes. The data suggest that vessels obtained from c-sections provide better contractility and superior prostacyclin release rates than those obtained from vaginal deliveries. In future, attention has to be paid to the mode of delivery when selecting vessels as a biomaterial for vascular tissue engineering. Experiments are currently under way in a perfusion model to investigate whether the observed differences persist or level out during tissue engineering procedures. 


\section{Acknowledgements}

The authors acknowledge the excellent technical assistance of C. Leykauf, S. Bergmann, and K. Bielenberg, as well as the help of the midwives in collecting the cord samples. We are indebted to O. Ortmann (OB/GYN, Klinik für Frauenheilkunde und Geburtshilfe, University of Regensburg) for providing additional umbilical cords. J. Burkhart (Blood Donor Service of the Bavarian Red Cross, Munich, Germany) kindly arranged the sample transports between the participating universities. We thank $H$. Ebensberger for creating the scanning electron microscopy images. The study was funded by the Government of Bavaria as part of the STEMMAT project.

\section{References}

[1] Bordenave L, Fernandez P, Rémy-Zolghadri M, Villars S, Daculsi R, Midy D In vitro endothelialized ePTFE prostheses: clinical update 20 years after the first realization. Clin Hemorheol Microcirc 2005;33(3):227-34.

[2] Salacinski HJ, Goldner S, Giudiceandrea A, Hamilton G, Seifalian AM, Edwards A, et al. The mechanical behavior of vascular grafts: a review. J Biomater Appl 2001;15(3):241-78.

[3] Badylak SF The extracellular matrix as a biologic scaffold material. Biomaterials 2007;28(25):3587-93.

[4] Hoenicka M, Lehle K, Jacobs VR, Schmid FX, Birnbaum DE Properties of the human umbilical vein as a living scaffold for a tissue-engineered vessel graft. Tissue Eng 2007;13(1):219-29.

[5] Jacobs VR, Niemeyer M, Gottschalk N, Schneider KTM, Kiechle M, Oostendorp RAJ, et al. Das STEMMAT-Projekt als Teil der Gesundheitsinitiative BayernAktiv: Adulte Stammzellen aus Nabelschnur und -blut als Alternative zur embryonalen Stammzellforschung [The STEMMAT project as part of the health initiative BayernAktiv: adult stem cells from umbilical cord and cord blood as alternative to embryonic stem cell research]. Zentralbl Gynakol 2005;127(6):368-72.

[6] Daniel J, Abe K, McFetridge PS Development of the human umbilical vein scaffold for cardiovascular tissue engineering applications. ASAIO J 2005;51(3):252-61. 
[7] Hoerstrup SP, Zünd G, Sodian R, Schnell AM, Grünenfelder J, Turina MI Tissue engineering of small caliber vascular grafts. Eur J Cardiothorac Surg 2001;20(1):164-9.

[8] Pence S, Kocoglu H, Balat O, Balat A The effect of delivery on umbilical arterial cord blood gases and lipid peroxides: comparison of vaginal delivery and cesarean section. Clin Exp Obstet Gynecol 2002;29(3):212-4.

[9] Rogers MS, Mongelli JM, Tsang KH, Wang CC, Law KP Lipid peroxidation in cord blood at birth: the effect of labour. $\mathrm{Br} \mathrm{J}$ Obstet Gynaecol 1998;105(7):739-44.

[10] Raijmakers MTM, Roes EM, Steegers EAP, van der Wildt B, Peters WHM Umbilical glutathione levels are higher after vaginal birth than after cesarean section. J Perinat Med 2003;31(6):520-2.

[11] Miller NM, Fisk NM, Modi N, Glover V Stress responses at birth: determinants of cord arterial cortisol and links with cortisol response in infancy. BJOG 2005;112(7):921-6.

[12] Aisien AO, Towobola OA, Otubu JA, Imade GE Umbilical cord venous progesterone at term delivery in relation to mode of delivery. Int J Gynaecol Obstet 1994;47(1):27-31.

[13] Jouppila R, Puolakka J, Kauppila A, Vuori J Maternal and umbilical cord plasma noradrenaline concentrations during labour with and without segmental extradural analgesia, and during caesarean section. Br J Anaesth 1984;56(3):251-5.

[14] Boelsterl M Der prädiktive Wert perinataler fetaler und maternaler Geburtsparameter für den relativen Gehalt mononukleärer und CD34-positiver Zellen im Nabelschnurblut Neugeborener [The predictive value of perinatal fetal and maternal birth parameters for the concentration of mononuclear and CD34-positive cells in cord blood] (Thesis). Munich: Technische Universität München, 2007.

[15] Li W, Huang T, Zeng Y, Yao $Z$ The relationship between gestational age and compliance in human umbilical vein and its possible application in vascular grafting. Ann Vasc Surg 2006;20(2):237-42.

[16] Lehle K, Hoenicka M, Jacobs VR, Schmid FX, Birnbaum DE Identification and reduction of cryoinjury in endothelial cells: a 
first step towards establishing a cell bank for vascular tissue engineering. Tissue Eng 2006;12(12):3439-47.

[17] Goldkrand JW, Schulte RL, Messer RH Maternal and fetal plasma cortisol levels at parturition. Obstet Gynecol 1976;47(1):41-5.

[18] Falconer AD, Poyser LM Fetal sympatho-adrenal mediated metabolic responses to parturition. $\mathrm{Br} \mathrm{J}$ Obstet Gynaecol 1986;93(7):747-53.

[19] Facchinetti F, Lanzani A, Genazzani AR Fetal intermediate lobe is stimulated by parturition. Am J Obstet Gynecol 1989;161(5):1267-70.

[20] Irestedt L, Dahlin I, Hertzberg T, Sollevi A, Lagercrantz H Adenosine concentration in umbilical cord blood of newborn infants after vaginal delivery and cesarean section. Pediatr Res 1989;26(2):106-8.

[21] Bird JA, Spencer JA, Mould T, Symonds ME Endocrine and metabolic adaptation following caesarean section or vaginal delivery. Arch Dis Child Fetal Neonatal Ed 1996;74(2):F132-F134.

[22] Gitau R, Menson E, Pickles V, Fisk NM, Glover V, MacLachlan N Umbilical cortisol levels as an indicator of the fetal stress response to assisted vaginal delivery. Eur J Obstet Gynecol Reprod Biol 2001;98(1):14-7.

[23] Marshall NJ, Goodwin CJ, Holt SJ A critical assessment of the use of microculture tetrazolium assays to measure cell growth and function. Growth Regul 1995;5(2):69-84.

[24] Berridge MV, Herst PM, Tan AS Tetrazolium dyes as tools in cell biology: new insights into their cellular reduction. Biotechnol Annu Rev 2005;11127-52.

[25] Pennati G Biomechanical properties of the human umbilical cord. Biorheology 2001;38(5-6):355-66.

[26] Furchgott RF, Cherry PD, Zawadzki JV, Jothianandan D Endothelial cells as mediators of vasodilation of arteries. J Cardiovasc Pharmacol 1984;6 Suppl 2S336-S343.

[27] Altura BM, Malaviya D, Reich CF, Orkin LR Effects of vasoactive agents on isolated human umbilical arteries and veins. Am J Physiol 1972;222(2):345-55. 
[28] Quan A, Leung SWS, Lao TT, Man RYK 5-hydroxytryptamine and thromboxane A2 as physiologic mediators of human umbilical artery closure. J Soc Gynecol Investig 2003;10(8):490-5.

[29] Rogines-Velo MP, Pelorosso FG, Zold CL, Brodsky PT, Rothlin RP Characterization of 5-HT receptor subtypes mediating contraction in human umbilical vein. 2. Evidence of involvement of 5-HT1B receptors using functional studies. Naunyn Schmiedebergs Arch Pharmacol 2002;366(6):596-604.

[30] Rogines-Velo MP, Pelorosso FG, Zold CL, Nowak W, Pesce GO, Sardi SP, et al. Characterization of 5-HT receptor subtypes mediating contraction in human umbilical vein. 1. Evidence of involvement of 5-HT2A receptors using functional and radioligand binding assays. Naunyn Schmiedebergs Arch Pharmacol 2002;366(6):587-95.

[31] Karlsson C, Bodelsson G, Bodelsson M, Stjernquist M Characterization of 5-hydroxytryptamine receptors mediating circular smooth muscle contraction in the human umbilical artery. Gynecol Obstet Invest 1999;47(2):102-7.

[32] Fecteau KA, Eiler H Placenta detachment: unexpected high concentrations of 5-hydroxytryptamine (serotonin) in fetal blood and its mitogenic effect on placental cells in bovine. Placenta 2001;22(1):103-10.

[33] Varela AF, Runge A, Ignarro LJ, Chaudhuri G Nitric oxide and prostacyclin inhibit fetal platelet aggregation: a response similar to that observed in adults. Am $\mathrm{J}$ Obstet Gynecol 1992;167(6):1599-604.

[34] Moncada S, Vane JR The role of prostacyclin in vascular tissue. Fed Proc 1979;38(1):66-71.

[35] Klockenbusch W, Braun MS, Schröder H, Heckenberger RE, Strobach H, Schrör K Prostacyclin rather than nitric oxide lowers human umbilical artery tone in vitro. Eur J Obstet Gynecol Reprod Biol 1992;47(2):109-15.

[36] Cruz MA, Gallardo V, Miguel P, Carrasco G, González C Mediation by 5-HT2 receptors of 5-hydroxytryptamine-induced contractions of human placental vein. Gen Pharmacol 1998;30(4):483-8.

[37] Williams KI, El Tahir KE Effects of uterine stimulant drugs on 
prostacyclin production by the pregnant rat myometrium. I. Oxytocin, bradykinin and PGF2 alpha. Prostaglandins 1980;19(1):31-8.

[38] Goyal A, Wang Y, Su H, Dobrucki LW, Brennan M, Fong P, et al. Development of a model system for preliminary evaluation of tissue-engineered vascular conduits. J Pediatr Surg 2006;41(4):787-91.

[39] Dardik H, Wengerter K, Qin F, Pangilinan A, Silvestri F, Wolodiger $\mathrm{F}$, et al. Comparative decades of experience with glutaraldehydetanned human umbilical cord vein graft for lower limb revascularization: an analysis of 1275 cases. J Vasc Surg 2002;35(1):64-71.

[40] Lamm P, Juchem G, Milz S, Schuffenhauer M, Reichart B Autologous endothelialized vein allograft: a solution in the search for small-caliber grafts in coronary artery bypass graft operations. Circulation 2001;104(12 Suppl 1):I108-I114. 


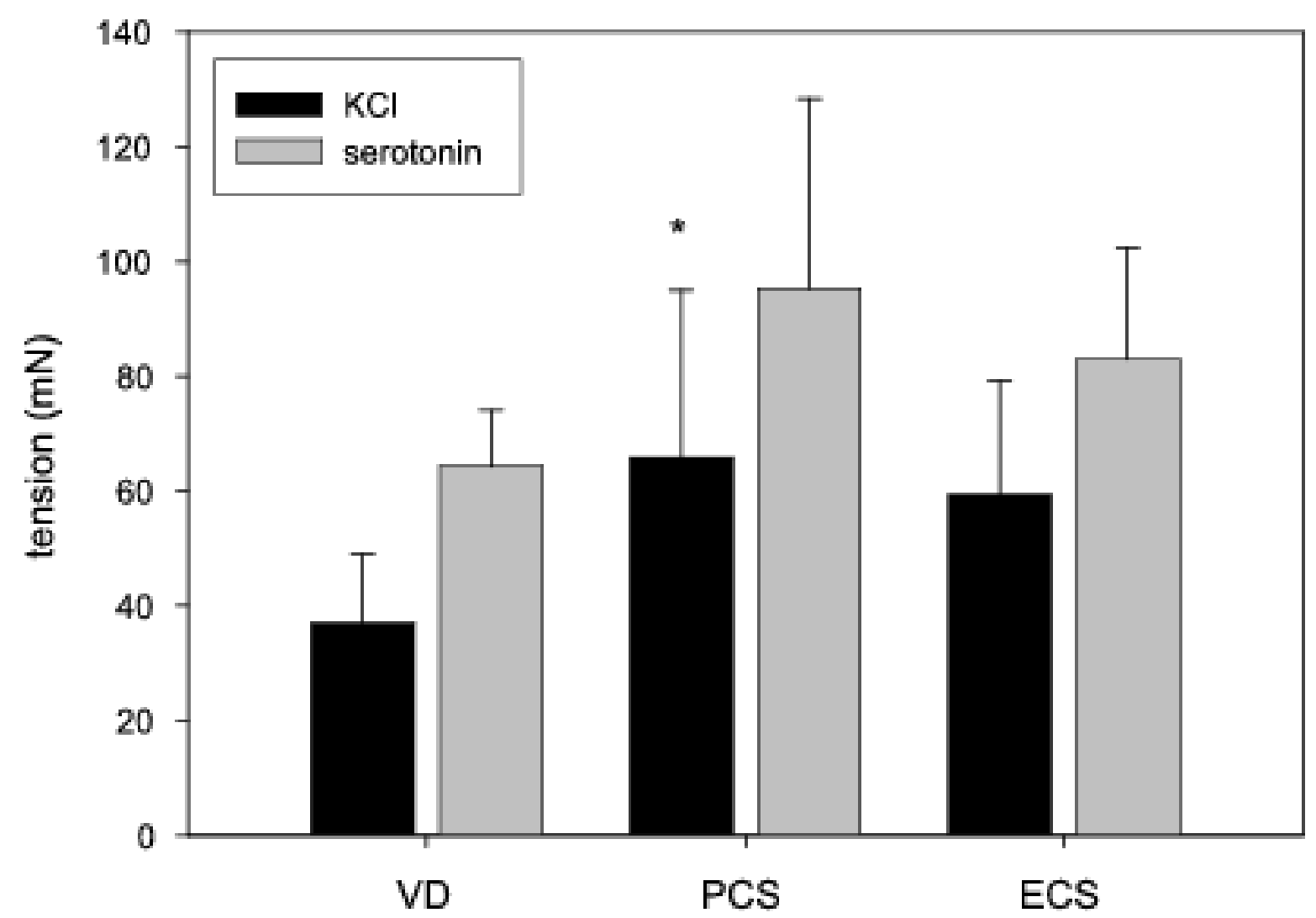

Figure 1: Comparison of the maximum tension of umbilical veins after depolarizing the vessel rings with potassium chloride (black bars) and after administering serotonin (grey bars) between vaginal delivery (VD, $n=9)$, planned $c-$ section (PCS, $n=8$ ), and emergency $c$-section (ECS, $n=6)$. * significantly different from VD (ANOVA, $p=0.029$ ). 

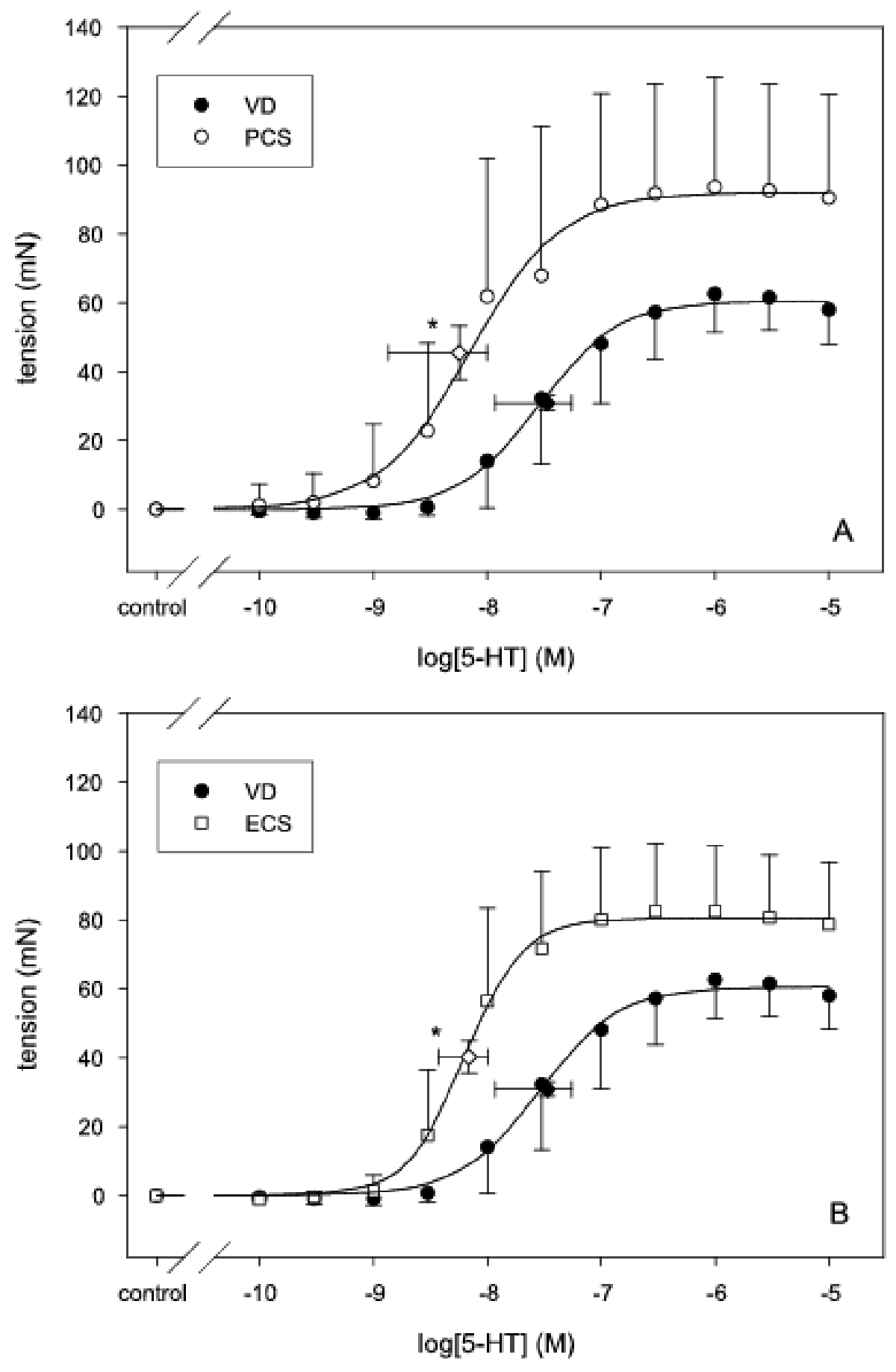

Figure 2: Serotonin dose-response curves of umbilical vein rings obtained from planned c-sections (PCS; $n=8$, open circles, panel $A$ ) and from emergency c-sections (ECS; $n=6$, open squares, panel $B$ ). Both are compared against the curve obtained with rings from vaginal deliveries (VD; $n=9$, closed circles). The $E_{50}$ values of each curve were calculated using nonlinear regression and are indicated as diamonds. * ${ }^{*} \mathrm{E}_{50}$ values are significantly different from 
VD (ANOVA, $p<0.05)$
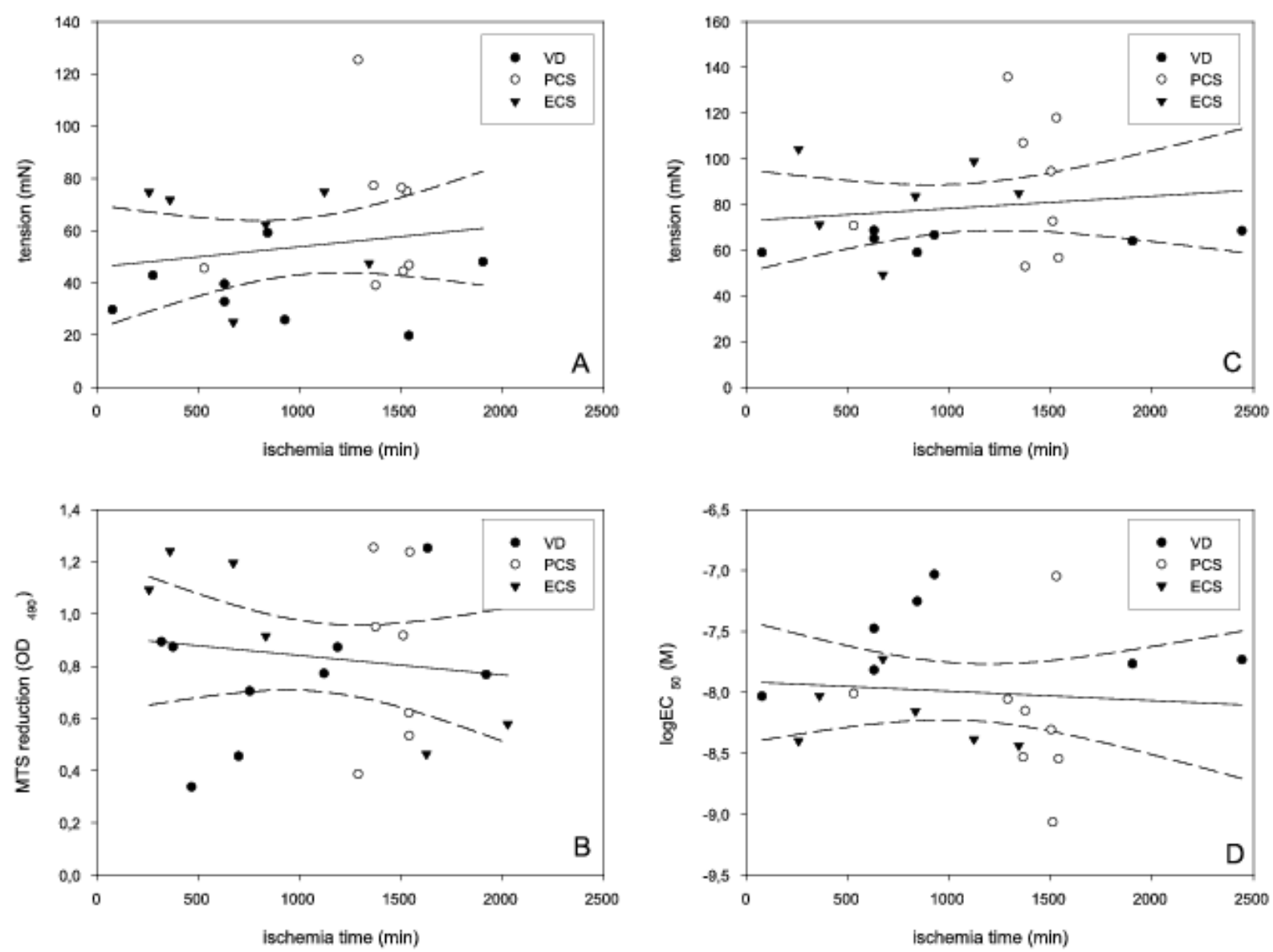

Figure 3: Lack of correlation of the maximum constriction by potassium chloride (A), of the MTS reduction (B), of the maximum constriction by serotonin $(C)$, and of the $\mathrm{EC}_{50}$ of serotonin (D) with the duration of ischemia. Filled circles, vaginal deliveries (VD); open circles, planned c-sections (PCS), filled triangles, emergency c-sections (ECS). The solid lines indicate the linear regressions of all samples regardless of the birth mode, the dashed lines indicate the 95\% confidence intervals. 


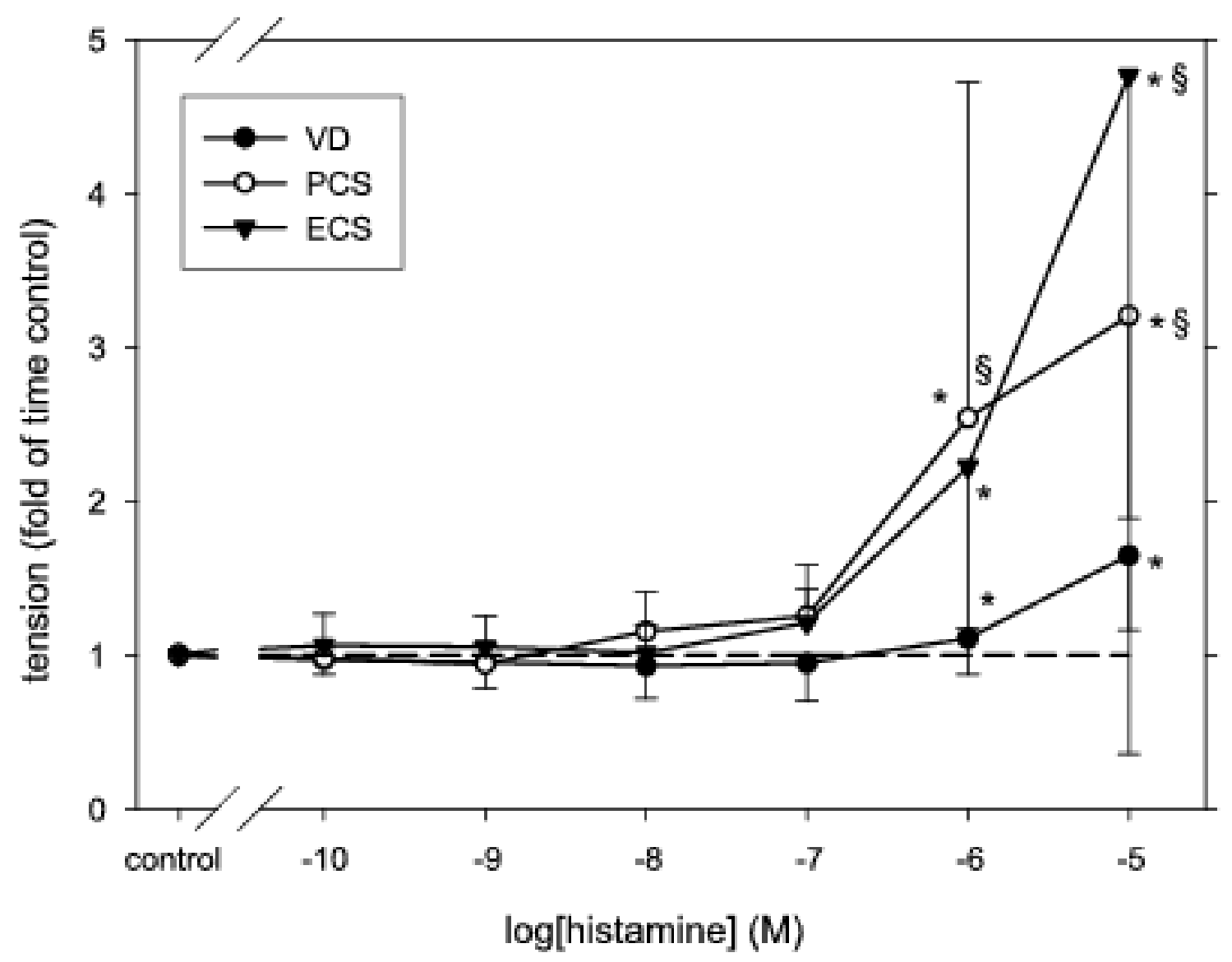

Figure 4: Histamine dose-response curves of umbilical vein rings obtained from planned c-sections (PCS; $n=5$, open circles), from emergency c-sections (ECS; $n=7$, filled triangles), and from vaginal deliveries (VD; $n=7$, closed circles). The values are plotted as the ratio of the tension after Histamine adminstration and the tension of matched time controls. Values less than unity (dashed line) indicate a relaxation, values higher than unity a constriction. * significantly different from unity (two-way repeated measurements ANOVA, $p<0.05$ ). $\S$ significantly different from VD (two-way ANOVA, $p=0.042$ ). 


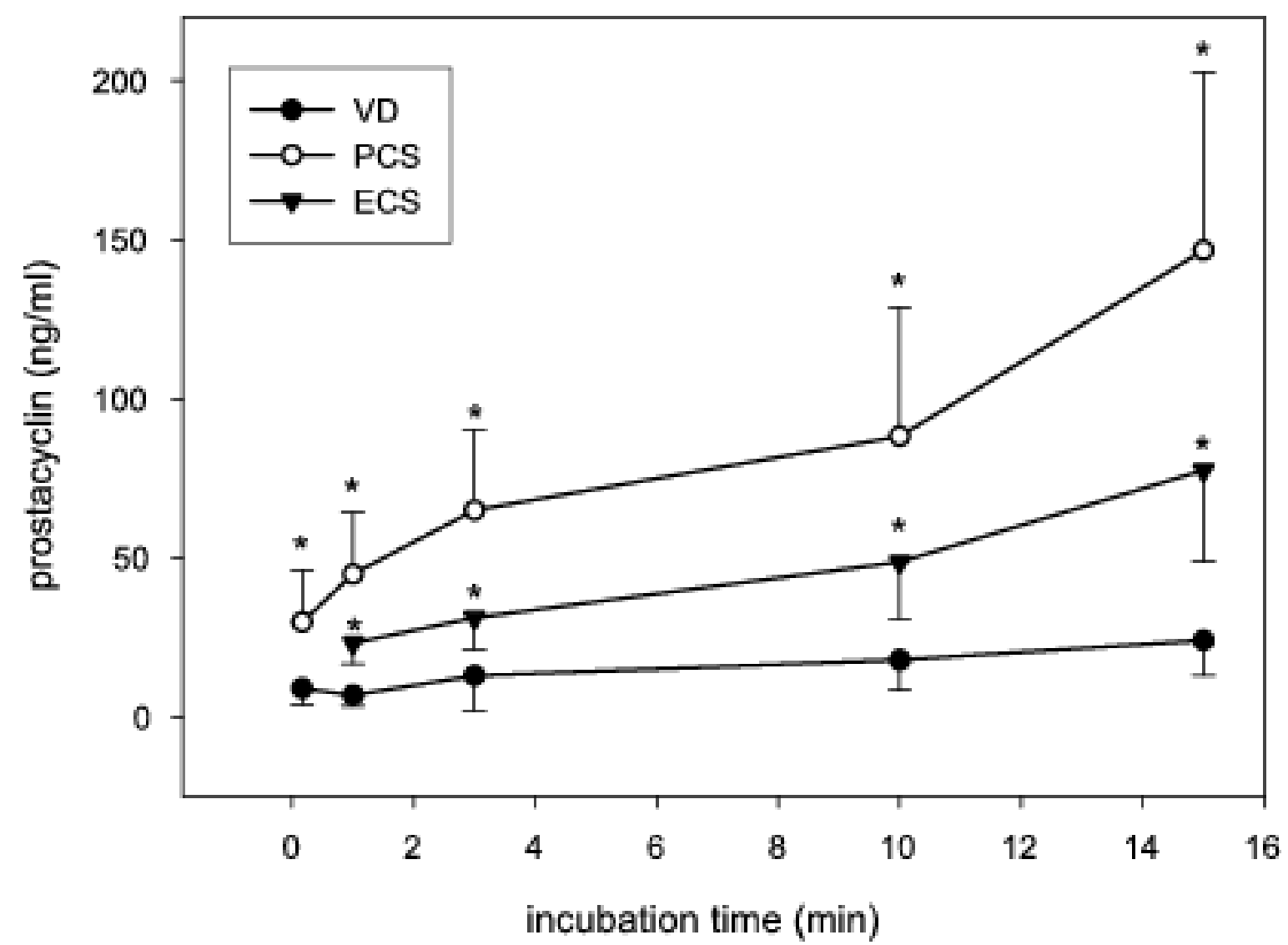

Figure 5: Time-dependent prostacyclin release from umbilical veins obtained from planned c-sections (PCS; $n=7$, open circles), from emergency $c$-sections (ECS; $n=6$, filled triangles), and from vaginal deliveries (VD; $n=10, c l o s e d$ circles). * significantly different from VD (two-way ANOVA, $p<0.001$ ). 
Umbilical vein function and birth mode
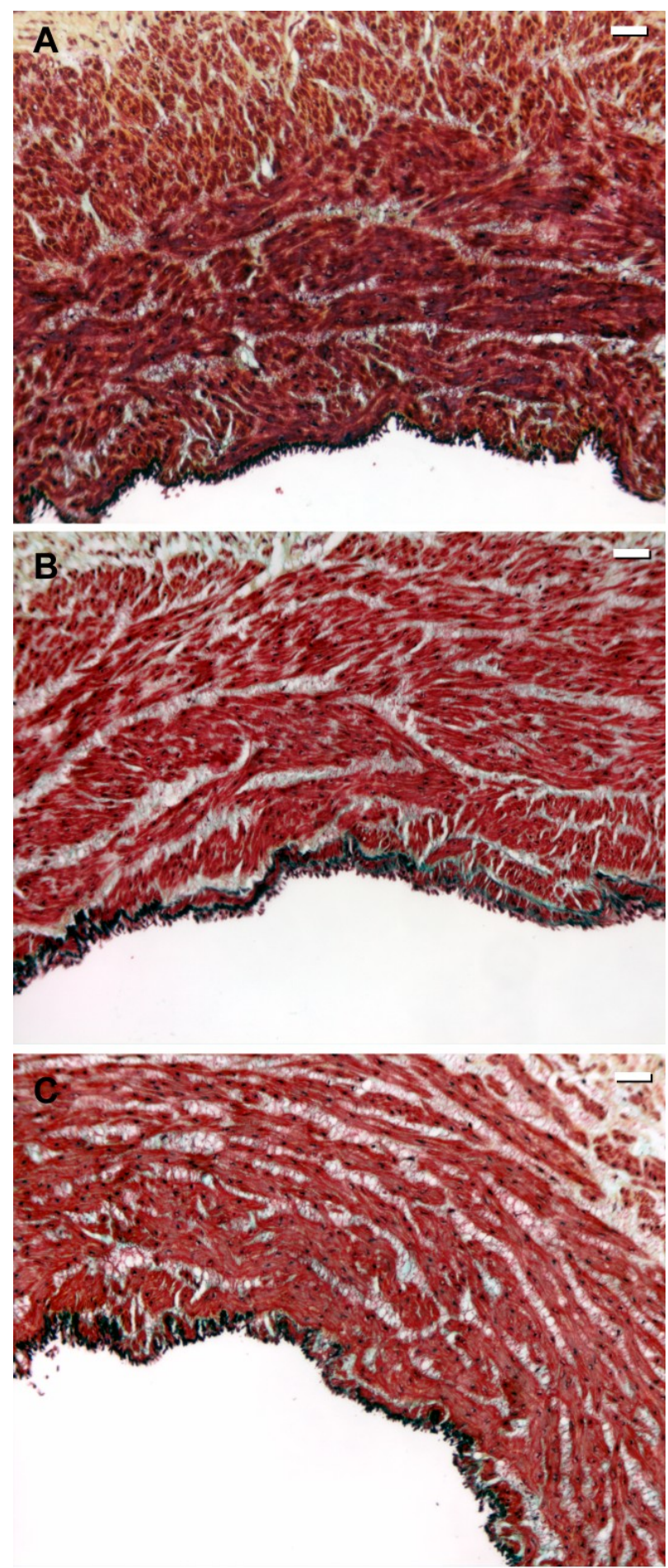

Figure 6: Comparison of HUV cross sections (Russel-Movat Pentachrome stain) of vaginal deliveries (A), primary csections (B), and emergency c-sections (C). The images are representative for 6 vessels per birth mode. The bars indicate $50 \mu \mathrm{m}$. 


\begin{tabular}{|c|c|c|c|}
\hline parameter & vaginal delivery & planned c-section & emergency c-section \\
\hline number (male / female) & $40(19 / 21)$ & $19(7 / 12)$ & $18(10 / 8)$ \\
\hline maternal age $(\mathrm{y})$ & $29.8(21-38)$ & $31.7(25-38)$ & $33.1(26-43)$ \\
\hline gestational age $(d)$ & $277.6(251-293)$ & $267.1(258-285)$ * & $275.1(259-288)$ \\
\hline birth weight (g) & $3487.8(2500-4470)$ & $2873.8(2025-3730){ }^{\S}$ & $3108.9(2620-3550)$ \\
\hline arterial cord blood $\mathrm{pH}$ & $7.24(7.08-7.42)$ & $7.28(7.14-7.39)$ & $7.26(7.09-7.34)$ \\
\hline Apgar score 1' & $8.65(6-10)$ & $8.28(7-9)$ & $7.94(5-10)$ \\
\hline Apgar score $5^{\prime}$ & $9.68(8-10)$ & $9.06(8-10) \S$ & $9.11(8-10){ }^{\S}$ \\
\hline Apgar score 10' & $9.84(9-10)$ & $9.28(8-10){ }^{\S}$ & $9.44(9-10)^{\S}$ \\
\hline ischemia time (min) & $1009(277-2443)$ & $1465(77-1543){ }^{\ddagger}$ & $1203(258-2123)$ \\
\hline
\end{tabular}

Table 1: Birth parameters of the umbilical cords used in this study. Except for the number of births, all data are reported as mean (range). Ischemia time is reported as median (range). ${ }^{*}$ significantly different from vaginal delivery and from emergency c-section (ANOVA, $p<0.001$ ); $\S$ significantly different from vaginal delivery $(A N O V A, p<0.001)$; $\ddagger$ significantly different from vaginal delivery (ANOVA, $p=0.043$ ). 